\title{
Quantitative and qualitative analysis of bone flap resorption in patients undergoing cranioplasty after decompressive craniectomy
}

\author{
Tommi K. Korhonen, MBBS, ${ }^{1}$ Niina Salokorpi, MD, PhD, ${ }^{1}$ Jaakko Niinimäki, MD, PhD, ${ }^{2}$ \\ Willy Serlo, MD, PhD, ${ }^{3,5}$ Petri Lehenkari, MD, PhD, ${ }^{4}$ and Sami Tetri, MD, PhD ${ }^{1}$

\begin{abstract}
1Department of Neurosurgery, Oulu University Hospital; ${ }^{2}$ Research Unit of Medical Imaging, Physics and Technology; ${ }^{3}$ PEDEGO Research Unit; and ${ }^{4}$ Department of Anatomy and Cell Biology, University of Oulu; and ${ }^{5}$ Department of Children and Adolescents, Oulu University Hospital, MRC Oulu, Finland
\end{abstract}

OBJECTIVE Autologous bone cranioplasty after decompressive craniectomy entails a notable burden of difficult postoperative complications, such as infection and bone flap resorption (BFR), leading to mechanical failure. The prevalence and significance of asymptomatic BFR is currently unclear. The aim of this study was to radiologically monitor the longterm bone flap survival and bone quality change in patients undergoing autologous cranioplasty.

METHODS The authors identified all 45 patients who underwent autologous cranioplasty at Oulu University Hospital, Finland, between January 2004 and December 2014. Using perioperative and follow-up CT scans, the volumes and radiodensities of the intact bone flap prior to surgery and at follow-up were calculated. Relative changes in bone flap volume and radiodensity were then determined to assess cranioplasty survival. Sufficient CT scans were obtainable from $41(91.1 \%)$ of the 45 patients.

RESULTS The 41 patients were followed up for a median duration of 3.79 years (25th and 75 th percentiles $=1.55$ and 6.66). Thirty-seven (90.2\%) of the 41 patients had some degree of BFR and $13(31.7 \%)$ had a remaining bone flap volume of less than $80 \%$. Patients younger than 30 years of age had a mean decrease of $15.8 \%$ in bone flap volume compared with the rest of the cohort. Bone flap volume was not found to decrease linearly with the passing of time, however. The effects of lifestyle factors and comorbidities on BFR were nonsignificant.

CONCLUSIONS In this study BFR was a very common phenomenon, occurring at least to some degree in $90 \%$ of the patients. Decreases in bone volume were especially prominent in patients younger than 30 years of age. Because the progression of resorption during follow-up was nonlinear, routine follow-up CT scans appear unnecessary in monitoring the progression of BFR; instead, clinical follow-up with mechanical stability assessment is advised. Partial resorption is most likely a normal physiological phenomenon during the bone revitalization process.

https://thejns.org/doi/abs/10.3171/2017.8.JNS171857

KEY WORDS autograft; bone resorption; cranioplasty; graft survival

$\mathrm{D}$ ECOMPRESSIVE craniectomy is the tertiary option for treating increased intracerebral pressure that is resistant to conservative treatment. Most of the evidence for its usefulness concerns malignant middle cerebral artery infarction leading to cerebral edema; ${ }^{42}$ other indications include hemorrhagic stroke ${ }^{12}$ and head trauma. ${ }^{20}$ Regardless of the etiology, the cranial bone defect produced in decompressive craniectomy warrants later repair in cranioplasty.

Autologous bone has traditionally been considered the preferred material for primary cranioplasty whenever available, ${ }^{4,14,19,37}$ being superior to synthetic materials mainly in terms of its guaranteed bio-, histo-, and immunocompatibility ${ }^{44}$ and the presumed cost-effectiveness of its use. The structure is also usually a perfect fit to the recipient site, except in the case of trauma. In addition, the long-term viability of synthetic implants has been a source of concern. ${ }^{15,22,25,27}$ Complications are common after cranioplasties both with autologous bone and when using synthetic implants, and can occur in both immediate and late

ABBREVIATIONS BFR = bone flap resorption; $\mathrm{HU}=$ Hounsfield unit; IBF = initial bone flap; $\mathrm{RBV}=$ remaining bone volume .

SUBMITTED July 29, 2017. ACCEPTED August 21, 2017.

INCLUDE WHEN CITING Published online February 23, 2018; DOI: 10.3171/2017.8.JNS171857. 
postoperative phases. The main disadvantage of cryopreserved and thus devitalized ${ }^{3,31}$ bone flaps is that they are especially prone to late bone flap resorption (BFR), with prevalence rates between $1.4 \%$ and $32.0 \%{ }^{21,28}$ and even higher rates of up to $66.7 \%$ in pediatric populations. ${ }^{6,16,26,33}$ There are no known surgical techniques that have been shown to have any effect on BFR.

Because only limited quantitative and qualitative information is available on postcranioplasty BFR at present, we set out to assess BFR in terms of the change in radiographic bone flap volume and radiodensity. Similar CT-based volumetry techniques have previously been applied in other specialties, such as hepatology and surgical oncology, $1,13,23,39$ having been first introduced as early as 4 decades ago. ${ }^{7,18}$

The aim of the current study was to assess 1) the prevalence of BFR in patients with decompressive craniectomy repaired later with an autologous bone flap; 2) the risk factors for BFR; and most importantly, 3) the extent of BFR increase over time. To investigate these questions, we identified all patients treated with craniectomy and subsequent autologous bone cranioplasty in our hospital district and performed follow-up CT scans on these patients.

\section{Methods}

\section{Patients and Selection}

We identified all 45 patients who underwent primary autologous cranioplasty at Oulu University Hospital, Finland, between January 1, 2004, and December 31, 2014. Of these 45 patients, 41 (91.1\%) were included in the study and 4 (8.9\%) were excluded: 1 due to missing radiological data and $3(6.7 \%)$ because they had died before the required CT scans could be obtained. Minor cranioplasties (e.g., trepanation hole repairs) and cranioplasties for craniosynostoses were excluded from the material. Oulu University Hospital is a tertiary-level center and the only hospital with a neurosurgical department in northern Finland, giving it a catchment population of 741,950 as of December 2015. All patients in the catchment area requiring neurosurgical interventions are referred to Oulu University Hospital. The patient data were extracted retrospectively from the medical records.

The study was performed in accordance with the Declaration of Helsinki on ethical principles for medical research. This was a treatment outcome study that followed the established clinical protocols of Oulu University Hospital and did not require additional informed consent from the patients. The Ethics Review Committee of the Northern Ostrobothnia Hospital District approved the study protocol.

\section{Clinical Data}

Follow-up changes in bone volume and radiodensity were evaluated in relation to retrospective clinical data; the variables recorded included initial bone flap (IBF) volume, bone flap volume at follow-up, sex (male or female), age at operation, cranioplasty outcome (normal recovery vs implant removal), complications, date of death, fragmentation of the bone flap, presence of CSF diversion, and smoking habits. If smoking was not specifically mentioned in the patient records, the patient was considered to be a nonsmoker. Age groups were defined with cutoff values of 0-30 years and more than 30 years to facilitate comparison with other reports, as similar cutoffs have been used previously in the literature. ${ }^{26,36}$

Nontraumatic patients with a primary diagnosis of intracerebral hemorrhage, subarachnoidal hemorrhage, or intracranial ischemia were combined into one "stroke" group. Clinically significant BFR was defined as a lysis of the bone flap severe enough to require removal of the bone flap. Dates of death were provided by Statistics Finland.

\section{Radiological Methods}

\section{IBF Determination}

All patients had undergone CT scans either preoperatively or immediately postoperatively, or both, in which case the latter was used. If an immediate postoperative CT scan was not available, IBF was determined from the preoperative CT scan. In such cases the exact measurement of the bone flap size was taken from the follow-up CT scan and the measurement was overlaid on the preoperative CT scan to determine the bone flap size, from which the preoperative bone flap volume was calculated (Fig. 1).

The radiological bone flap status at this point will be referred to as the IBF. A postoperative scan was found and used for determining the volume of the IBF in 33 cases $(80.5 \%)$. Immediate postoperative scans were performed at a mean of 1.4 months after cranioplasty. An immediate postoperative scan suitable for IBF determination was found in the Oulu University Hospital database in 30 cases (73.2\%), and 3 others $(7.3 \%)$ were acquired from secondary centers. For 8 patients $(19.5 \%)$ the IBF had to be determined from the preoperative $\mathrm{CT}$ scan.

\section{Follow-Up CT Scans}

Follow-up CT scans were conducted for all patients in the cohort. Patients were invited either to Oulu University Hospital or to a secondary center for follow-up CT scans if the latest available CT scan was more than 1 year old. Follow-up scans were conducted at a mean interval of 52 months (range 2-139 months) after cranioplasty. The bone flaps in 7 patients (17.1\%) had been removed before the follow-up CT, so that the bone flap volume was calculated from the pre-removal CT image.

\section{Bone Flap Volumetry}

Bone flap areas in the axial plane were measured from the IBF and follow-up CT scans using a computer interface that allowed for manual outlining of the bone flap. Care was taken to make both measurements at exactly the same level on the bone flap. The bone flap area (A) inside the outline was measured and converted to square millimeters by means of an image analysis program. Bone flap volumes $(\mathrm{V})$ in cubic millimeters were calculated using the summation-of-area method, as $\mathrm{V}=\mathrm{dy} \times\left(\mathrm{A}_{1}+\mathrm{A}_{2}\right.$ $\left.+\ldots+A_{n}\right)$, in which $A_{1}, A_{2}, \ldots, A_{n}$ indicate single axial plane bone flap areas. The vertical distance (dy) between the $\mathrm{CT}$ slices chosen for area measurements was $10 \mathrm{~mm}$. The remaining bone volume (RBV) was calculated in relative terms as $\mathrm{RBV}=(\mathrm{Vf} / \mathrm{Vi}) \times 100 \%$, in which $\mathrm{Vf}$ and $\mathrm{Vi}$ 
are the bone flap volumes in the follow-up and initial CT scans, respectively. Thus, an RBV $\geq 100 \%$ indicates complete absence of BFR, and an RBV below $100 \%$ implies the presence of BFR.

\section{Bone Flap Radiodensity}

Bone flap radiodensity was measured in Hounsfield units (HUs). HUs are a quantitative measure of radiodensity. Water and air are assigned values of 0 and -1000 HUs, respectively. The HU of bone varies in relation to the structure of the bone. The mean radiodensity $(\mathrm{R})$ of the bone flap was calculated as follows:

$$
\begin{gathered}
\mathrm{R}=\left(\mathrm{A}_{1} \times \mathrm{HU}_{1}+\mathrm{A}_{2} \times \mathrm{HU}_{2}+\ldots+\mathrm{A}_{5} \times \mathrm{HU}_{5}\right) / \\
\left(\mathrm{A}_{1}+\mathrm{A}_{2}+\ldots+\mathrm{A}_{5}\right)
\end{gathered}
$$

in which $\mathrm{HU}_{n}$ is the average $\mathrm{HU}$ value of the respective axial bone area $A_{n}$. Axial measurements were taken from 5 corresponding levels on the bone flap area and the contralateral skull area.

To adjust for errors associated with the use of the Hounsfield scale as absolute values, ${ }^{5,24}$ relative values were used: the radiodensity of the bone flap was divided by the radiodensity of the adjacent untouched calvaria in both IBF and follow-up CT scans (Fig. 1). The change (dR) of this relative parameter in follow-up was calculated as $\mathrm{dR}=(\mathrm{Rf} / \mathrm{Ri}) \times 100 \%$, in which $\mathrm{Rf}$ and $\mathrm{Ri}$ are the relative radiodensities in follow-up and initial CT scans, respectively. Five patients $(12.2 \%)$ had received a bifrontal cranioplasty and were excluded from the radiodensity analyses due to missing untouched calvaria adjacent to the bone flap.

All CT scans were stored in a picture archiving and communication system. All radiological measurements were conducted by the first author (T.K.K.) using the image analysis program neaView Radiology (Neagen Ltd.).

\section{Bone Flap Cryopreservation}

According to our institutional protocol, after the craniectomy procedure meticulous sterility is retained and bacteriological culture samples are taken from the removed bone flaps. After obtaining the samples, the bone flap is sterilely double-sealed inside 2 dry sterile plastic bags and preserved with no cryoprotectants in $-70^{\circ} \mathrm{C}$ until cranioplasty. If the cultures taken are positive for any bacterium, the bone flap is disposed of and the future cranioplasty is conducted with a synthetic implant. Preceding the cranioplasty operation, the bone flap is allowed to thaw in a room-temperature saline solution. Until implantation, the bone flap has warmed up to room temperature.

\section{Statistical Analysis}

Continuous variables were analyzed with 1-way ANOVA, except where Levene's test for homogeneity of variances proved statistically significant, in which case the Brown-Forsythe test was applied instead. Variables were chosen for further linear regression analysis (the enter method) based on their clinical importance. For patients whose IBF was determined from an immediate postcranioplasty CT scan $(n=33)$, the follow-up duration was calculated as the time between the IBF CT scan and the follow-up CT scan. For patients whose IBF was determined from a precranioplasty CT scan $(n=8)$, the follow-up time was calculated as the time between cranioplasty and the follow-up CT scan. Medians with 25th and 75th percentiles or means with SDs are shown unless otherwise stated.

A linear regression model was constructed to estimate
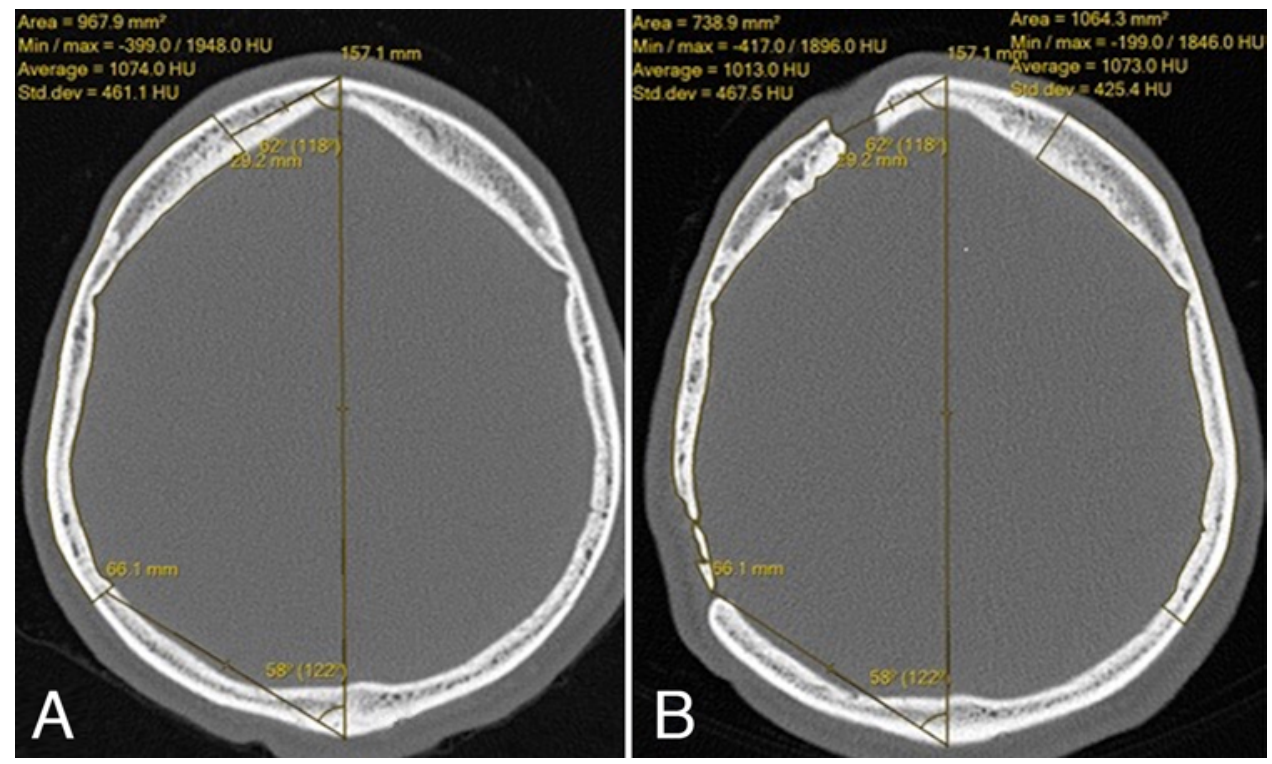

FIG. 1. CT scans depicting the manner in which axial bone flap areas and bone radiodensity in HUs were measured from the precranioplasty (intact bone flap, A) and postcranioplasty (follow-up, B) CT scans. The bone area inside the yellow outline represents a singular axial bone flap area as used in the bone volume and radiodensity calculations. In B, measurement of the contralateral bone area and radiodensity is also shown. The mean HUs of the outlined section are labeled as "Average." A decrease is seen in the HUs of the bone flap section compared with the contralateral bone area due to cavity formation in the flap. The remaining relative bone flap volume of this patient was $82.9 \%$. Figure is available in color online only. 
risk factors for decreased RBV. Because the impacts of age and follow-up time were nonlinear, these variables were categorized (age $<30$ years vs $\geq 30$ years, and follow-up duration by quartiles). A p value $<0.05$ was considered statistically significant. All the statistical analyses were performed with the Statistical Package for the Social Sciences (Windows version 22, SPSS, Inc.).

\section{Results}

\section{Descriptive Data}

Forty-one patients with autologous primary cranioplasty with a mean age at operation of $41 \pm 14.8$ years were included in the study. The ages ranged from 15 to 65 years. The $31(75.6 \%)$ male and $10(24.4 \%)$ female patients were followed up for a median duration of 3.79 years (25th and 75 th percentiles $=1.55$ and 6.66 ). The baseline characteristics and RBVs of these patients are shown in Table 1.

In all cases the primary etiology of the cranial defect had been surgical decompression with an emergency indication. The primary diagnoses were stroke in 24 cases (58.5\%), trauma in $16(39.0 \%)$, and an atraumatic subdural hematoma in $1(2.4 \%)$.

Follow-up CT scans were performed at a mean of $4.3 \pm$ 3.14 years after cranioplasty, with the dates ranging from 2 to 139 months postoperatively. The mean 2D lateral bone flap area was $85.4 \mathrm{~cm}^{2}$ (range 25.6-149.6 $\mathrm{cm}^{2}$ ). Sixteen patients $(39.0 \%)$ had one or more complications, and the bone flaps of 7 patients (17.1\%) had been removed. Cranioplasty removal operations were performed primarily due to BFR in 4 cases $(9.8 \%)$, while $3(7.3 \%)$ were primarily due to deep surgical site infection. Bacterial cultures were taken in all 4 cases with BFR, and an infection was found in 2 cases. The median time from cranioplasty to the BFR diagnosis in the group with BFR was 8.2 months (range $2.4-14.0$ months).

\section{Relative RBV and Radiodensity}

The RBVs of our patients are presented in Figs. 2 and 3. The mean RBV was $81.2 \% \pm 18.6 \%$ and the values of RBV ranged from $25.7 \%$ to $103.8 \%$. At least some BFR was present in $37(90.2 \%)$ of the 41 patients, i.e., RBV was below $100 \%$. RBV was less than $80 \%$ in 13 patients (31.7\%). In contrast, clinically significant BFR that required bone flap removal was found only in 4 patients $(9.8 \%)$. In patients with BFR that led to bone flap removal, the values of RBV ranged from $25.7 \%$ to $92.9 \%$ with a mean of $60.6 \%$ $\pm 36.7 \%$. Although this is less than the mean for all patients, statistical significance could not be reliably tested due to the small size of the BFR group. The mean RBV in the group of patients with all-cause bone flap removal was $76.2 \% \pm 32.6 \%$.

No associations between RBV and follow-up time, age, sex, smoking habits, indication for decompression, time interval between craniectomy and cranioplasty, fragmentation of the bone flap, or use of CSF-diverting shunts were found in the ANOVA analyses (Table 1).

The mean relative radiodensity in follow-up was $99.7 \%$ $\pm 11.3 \%$ when compared with the relative radiodensity in the IBF CT scans. The relative radiodensities ranged from $72.7 \%$ to $139.3 \%$.

\section{Effect of Follow-Up Duration and Patient Age on Remaining Bone Flap Volume}

To analyze whether BFR is a progressive phenomenon dependent on the follow-up duration, a linear regression model was constructed. The model included follow-up time and the patient's age (Table 2). The length of the follow-up time until measurement of the RBV was found to have no statistically significant effect on the RBV itself, but the mean RBV of patients younger than 30 years of age was $15.8 \%$ lower than that for the other age groups even though no linearity was found in the effect of age and follow-up duration.

TABLE 1. Characteristics of the primary autologous bone cranioplasty patients and their relative RBVs

\begin{tabular}{|c|c|c|c|}
\hline Characteristics & $\begin{array}{c}\text { Autologous } \\
\text { Cranioplasty } \\
\text { Patients }(n=41)^{*}\end{array}$ & $\begin{array}{c}\text { Mean } \\
\text { Relative RBV } \\
\%(S D) \dagger\end{array}$ & $\begin{array}{c}p \\
\text { Valuef }\end{array}$ \\
\hline Sex & & & 0.18 \\
\hline Male & $31(75.6)$ & $79.0(19.3)$ & \\
\hline Female & $10(24.4)$ & $88.1(15.1)$ & \\
\hline Age (yrs) & & & 0.08 \\
\hline $0-29$ & $11(26.8)$ & $70.0(27.0)$ & \\
\hline $30-49$ & $16(39.0)$ & $83.2(11.6)$ & \\
\hline$\geq 50$ & $14(34.1)$ & $87.9(13.5)$ & \\
\hline Bone flap status & & & 0.65 \\
\hline Removed & $7(17.1)$ & $76.2(32.6)$ & \\
\hline In place & $34(82.9)$ & $82.2(14.8)$ & \\
\hline Smoking habits & & & 0.17 \\
\hline Smoker & $14(34.1)$ & $86.7(13.0)$ & \\
\hline Nonsmoker & $27(65.9)$ & $78.3(20.5)$ & \\
\hline Dead§ & $3(7.3)$ & & \\
\hline Primary diagnosis & & & 0.19 \\
\hline Trauma & $16(39.0)$ & $76.5(23.5)$ & \\
\hline Stroke & $24(58.5)$ & $85.4(13.3)$ & \\
\hline Subdural hematomaף & $1(2.4)$ & 55.4 & \\
\hline Bone flap fragmentation & & & 0.69 \\
\hline Fragmented & $7(17.1)$ & $77.4(27.9)$ & \\
\hline Nonfragmented & $34(82.9)$ & $82.0(16.5)$ & \\
\hline Use of ventriculostomy & & & 0.77 \\
\hline In place & $14(34.1)$ & $82.4(19.9)$ & \\
\hline None & $27(65.9)$ & $80.6(18.2)$ & \\
\hline Use of CSF shunt & & & 0.37 \\
\hline In place & $6(14.6)$ & $74.8(27.7)$ & \\
\hline None & $35(85.4)$ & $82.3(16.9)$ & \\
\hline CE-CP interval (mos) & & & 0.16 \\
\hline $0-3$ & $8(19.5)$ & $83.6(16.6)$ & \\
\hline$>3-6$ & $11(26.8)$ & $72.0(24.6)$ & \\
\hline$>6$ & $22(53.7)$ & $84.9(14.7)$ & \\
\hline
\end{tabular}

$\mathrm{CE}-\mathrm{CP}=$ craniectomy-cranioplasty.

* Data given as number of patients (\%).

$\dagger$ Mean RBV in all patients was 0.81 (SD 0.19).

$\ddagger p$ values obtained with 1-way ANOVA or Brown-Forsythe test.

$\S$ All excluded due to insufficient radiological data.

If Excluded from Brown-Forsythe test due to only 1 patient $(n=1)$. 


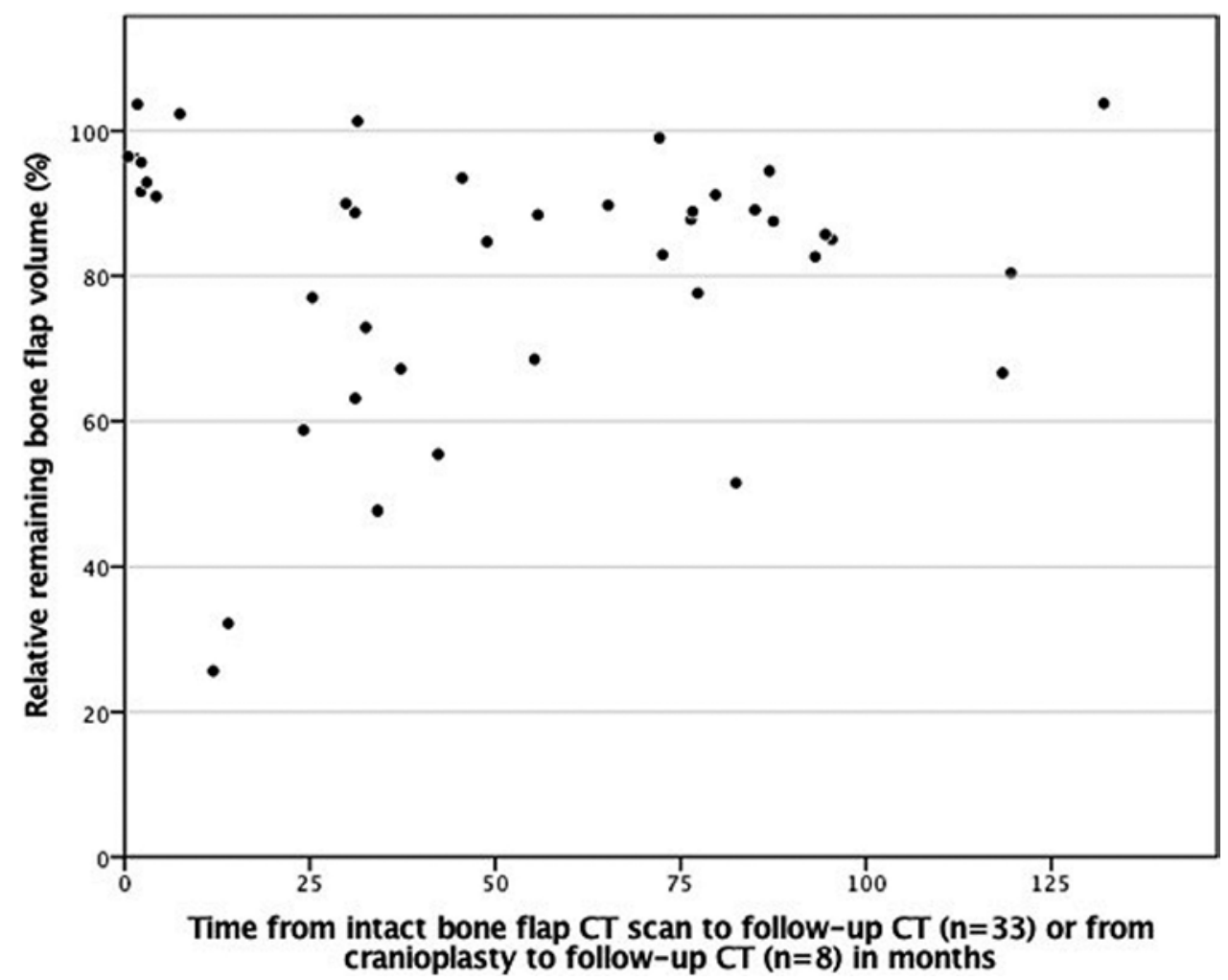

FIG. 2. Scatter plot showing the relative RBVs and follow-up durations of 41 autologous bone cranioplasty patients undergoing follow-up CT.

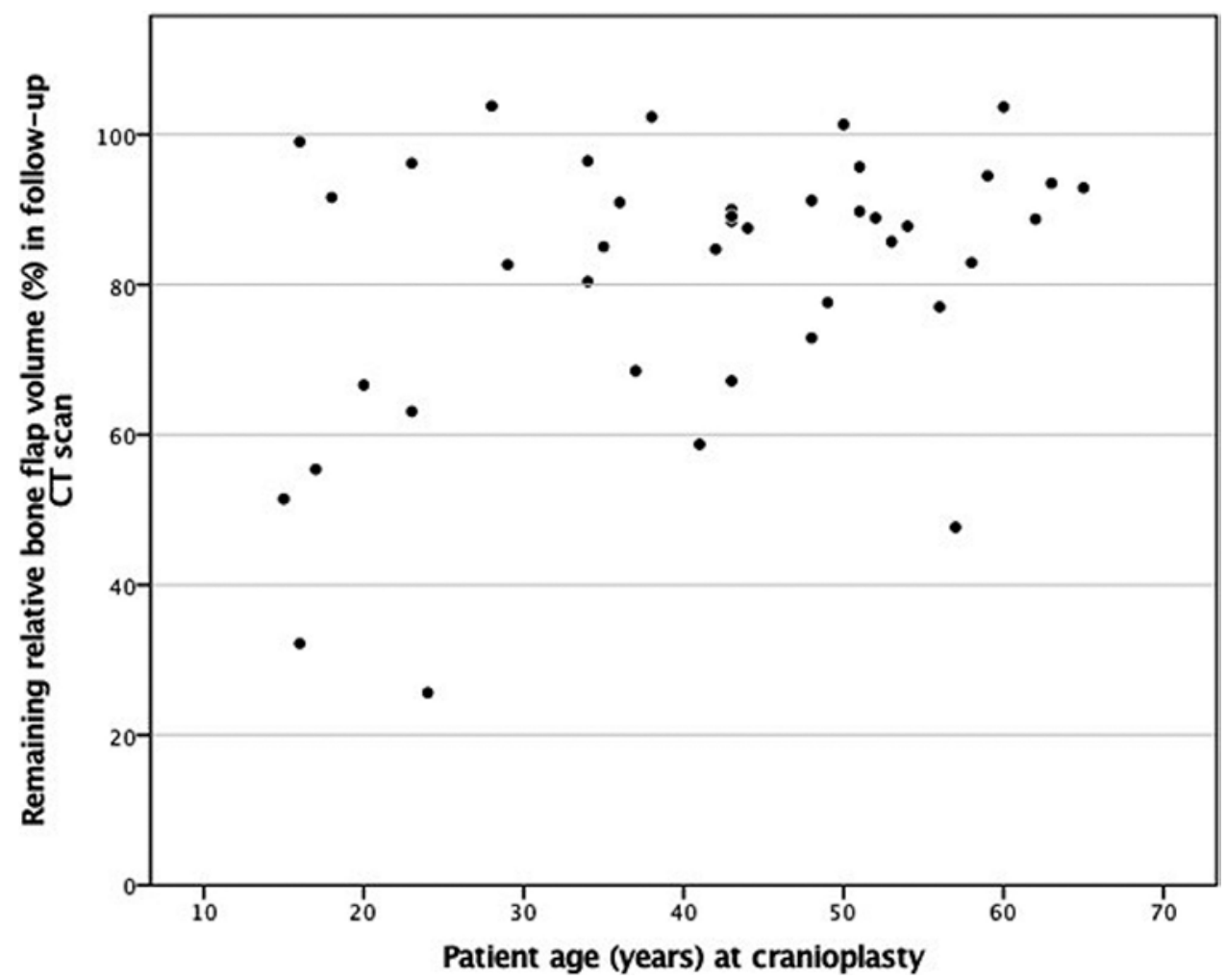

FIG. 3. Scatter plot showing the relative RBVs and ages of 41 autologous bone cranioplasty patients undergoing follow-up CT. 
TABLE 2. Results of the linear regression model used to assess the effect of follow-up time and patient age on relative RBV

\begin{tabular}{lccc}
\hline \multicolumn{1}{c}{ Variable } & Unstandardized B & $95 \% \mathrm{Cl}$ & $\mathrm{p}$ Value \\
\hline Constant & 0.70 & $0.56-0.84$ & $<0.01$ \\
\hline Follow-up time (wks) & & & \\
\hline 105-198 & -0.08 & -0.23 to 0.08 & 0.34 \\
\hline$>198$ to 346.6 & 0.01 & -0.15 to 0.17 & 0.90 \\
\hline$>346.6$ & 0.03 & -0.13 to 0.18 & 0.73 \\
\hline Age $>30$ yrs $^{*}$ & 0.17 & $0.03-0.30$ & 0.02 \\
\hline
\end{tabular}

$\mathrm{B}=$ regression coefficient; $\mathrm{Cl}=$ confidence interval.

* $p$ value $<0.05$ obtained with linear regression (the enter method).

\section{Discussion}

A successful cranioplasty and mechanical stabilization of the skull restores the natural cosmesis and cerebral protection while simultaneously contributing to improvements of the cerebral blood flow ${ }^{17}$ and neurocognitive outcome, ${ }^{10,38}$ which is an especially prominent factor in patients with sinking skin flap syndrome and associated neurological deteriorations. ${ }^{34}$ Postoperative complications are nevertheless common, ${ }^{41,43,44}$ which is also prevalent in our results: $39.0 \%$ of our patients had clinical complications and the implant removal rate was $17.1 \%$.

BFR, a late complication in which the bone flap fails to reintegrate and adhere to the cranium, is unique to autologous cranioplasties. The bone flap weakens, becoming loose and palpably soft. From the standpoint of clinical orthopedics, the resulting instability per se is a negative prognostic factor for ossification and, hence, the preservation of mechanical stability is essential. A major lack of bone union with the calvaria may compromise intraosseal blood flow into the bone flap and further prevent healing and reintegration. This warrants removal of the weakened bone flap and a subsequent cranioplasty with a synthetic implant, which in turn entails an additional exposure to complications. Another viewpoint is that it is not clear how and if some bone changes adjacent to fixation screws and plates concern resorption or its development. Thus, the mechanism behind the bone loss should be studied further to develop techniques to prevent it.

So far there are no standardized methods for measuring postcranioplasty BFR, and the qualitative analysis methods presented thus far ${ }^{11,36,41,45}$ include a degree of subjectivity. In this study we use a new method for evaluating bone volume from the bone sequence of CT scans to address this question quantitatively. Although similar methods have been successfully applied in other medical specialties, ${ }^{1,7,13,18,23,39}$ to the authors' knowledge this method has not previously been used for cranioplasty bone flap volumetry.

\section{Main Results}

The present results show a decrease in bone flap volume in more than $90 \%$ of our patients after autologous cranioplasty for a large-sized defect $\left(>25 \mathrm{~cm}^{2}\right)$ following emergency decompressive craniectomy. Thus, sterile BFR appears to be more common than was previously believed, as the majority of our patients had a decrease in relative $\mathrm{RBV}$, but a smaller percentage of patients required a repeat cranioplasty due to BFR. Consistent with these findings, we also found that follow-up time does not influence the severity of RBV decrease. BFR is nevertheless the most evident underlying cause for bone flap loosening and disintegration.

The fact that some degree of resorption and even structural bone loss occurred in the majority of cases raises the question of whether BFR is a time-dependent process leading to a higher incidence of clinically significant BFR as the follow-up time increases. According to the results obtained with our patient cohort having a median follow-up duration of 3.79 years, bone flap volume does not decrease linearly as a function of time. Thus, a decrease in bone volume at some point after cranioplasty does not necessarily indicate that the patient will require replacement of the bone flap due to its failure later. Instead, a moderate extent of BFR is likely a normal physiological phenomenon during the bone revitalization process.

In clinical practice, this means that there is no need for routine follow-up CT scans after the immediate recovery period to monitor BFR. Instead, clinical follow-up is likely to be sufficient if no additional symptoms are present. However, bearing in mind the tendency for our patients less than 30 years old at cranioplasty to have a lower RBV, and in light of previous results regarding patient age and $\mathrm{BFR}, 9,11,19,26,32,35$ the authors recommend that younger patients should be monitored more frequently after autologous cranioplasty.

Although the mean RBV of patients younger than 30 years was lower than that of the rest of the cohort $(69.8 \%$ vs $85.6 \%$ ), only borderline significance was reached in ANOVA. This is at least partially due to the age range of the cohort, with the youngest patient being 15 years old, whereas children under the age of 7 years have the maximum risk of BFR. ${ }^{26}$ Although no time-related linearity was found in this effect in the linear regression analysis, patients under 30 years of age had a clearly lower mean $\mathrm{RBV}$, so that younger age may predict lower RBV, but this is independent of the follow-up duration and it is likely that some other age-associated factor may expose younger patients to BFR.

Most cranioplasty failures in our patient cohort were due to BFR as there were 4 patients who presented with bone resorption that not only was clinically clear enough but also required a recranioplasty, and in no other cases was resorption severe enough to indicate repeat cranioplasty on clinical grounds or cause the issue to be brought up by the patients. Two patients presented with clinical manifestations of BFR more than a year (12.2 and 14.0 months) after cranioplasty, both of them males under 25 years of age (16 and 24 years), and both with an RBV below 33\% (25.7\% and 32.2\%; Fig. 4). Microbiological cultures were negative and no signs of infection were found. Thus, both patients underwent an undelayed repeat cranioplasty. In the rest of our cranioplasty patients the RBV was more than $47 \%$ (Figs. 2 and 3). Thus, an RBV less than $33 \%$ was enough to produce clear clinical findings of BFR in this cohort, but these results should be confirmed in a larger study with standardized clinical examinations of the surgical sites to find the minimum decrease in RBV required to produce clinically significant findings. 

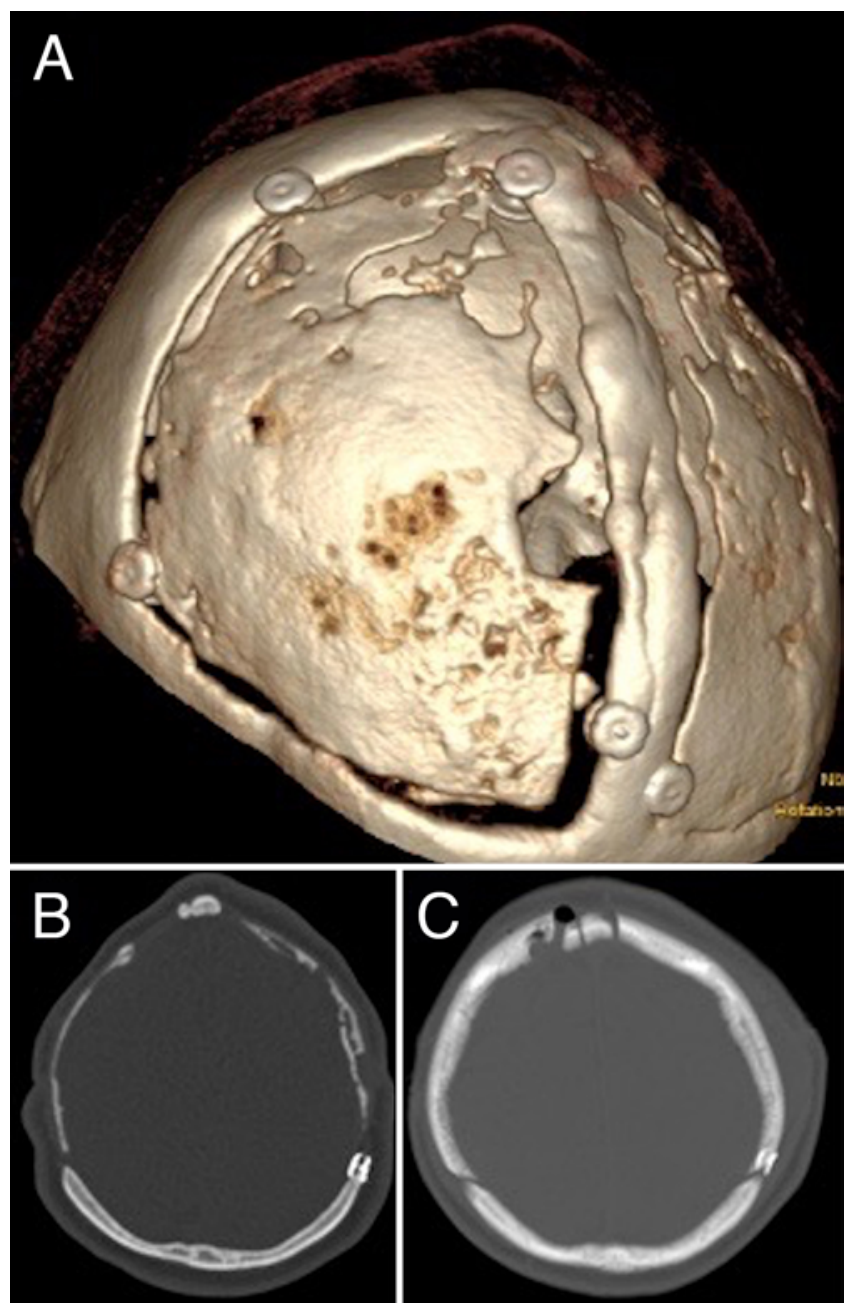

FIG. 4. Follow-up CT scans (A [3D reconstruction] and B) of a 24-yearold male patient taken exactly 1 year after bifrontal cranioplasty. The intact bone flap is illustrated in $\mathbf{C}$. An aseptic near-total lysis of the bone flap with a complete lack of bone union with the surrounding calvaria is noted in the follow-up. Diffuse thinning and numerous holes in the bone flap are also noted. The relative RBV of this patient was $25.7 \%$. The patient later underwent a secondary cranioplasty with a polyetheretherketone implant. Figure is available in color online only.

Wound infections were found in the remaining 2 patients with BFR, and softening and cortical irregularities of the bone flaps were discovered during the wound revision procedures. The bone flaps were therefore removed and delayed repeat cranioplasties were scheduled. These cases of BFR manifested clinically much sooner after cranioplasty (in 2.4 and 4.1 months). In spite of the clinical findings of infection, volumetric resorption was minimal, as RBV was more than $90 \%$ in both cases. We hypothesize that osteomyelitic inflammatory demineralization of the bone may have occurred in infected bone flaps ${ }^{2,29}$ and that the bone had thereby lost its integrity. Even so, the mineral components remain in the obliterated flap site, and bone-like material appears in CT. Thus, the measured RBV must have exaggerated the integrity of the bone flap in these cases. There is obviously a rationale for differentiating between infectious softening of the bone flap and sterile BFR, which appears to occur over a more prolonged period of time.

It has been found previously that shunt-dependent hydrocephalus is a risk factor for BFR, $6,11,30,36$ and the patients with hydrocephalus shunts in the present cohort did indeed tend to have a lower RBV (mean $74.8 \%$ vs $82.3 \%$ in the rest of the patients). Bone flap fragmentation has also been found previously to correlate with a higher risk of BFR, ${ }^{6,9,11,36}$ and correspondingly in the present series the mean RBV was slightly lower in the patients with fragmented bone flaps (77.4\%) than in those in whom the bone flap was intact (82.0\%). Because the subgroups consisted of only 6 and 7 patients, respectively, statistical significance was not achieved and no definite conclusions could be drawn. Additionally, no correlations were found between RBV and sex, smoking habit, indication for decompression, "freezer time" between craniectomy and cranioplasty, or fragmentation of the bone flap.

Four patients (9.8\%) had an RBV greater than $100 \%$ (with a maximum of 103.8\%). As these excesses are minor, they could in part be explained by measurement error. In addition, it was noted during RBV measurement that 15 patients $(36.6 \%)$ had signs of dural osteogenesis at the surgical sites under the bone flap (Fig. 5). Interestingly, some patients even had coinciding evidence of osteogenesis along with radiologically evident BFR. The role of the dura mater and the process of bone turnover at the histological level, and the association of these with the radiological and clinical findings following reimplantation of a frozen autologous bone flap, need further investigation and will be addressed in future publications.

The comparison of the relative radiodensities between follow-up and IBF CT scans showed notable variations of more than $66 \%$, thus confirming that the postreconstruction bone remains an area of high metabolic activity. Drawing more profound conclusions from the radiodensity analyses without a resorption grading system is difficult, as BFR has been noted to be a heterogenous complication with different types of lyses of the bone flaps ${ }^{45}$ For example, using the method described in this study, BFR characterized only by thinning of the bone flap with no significant resorption of the cortices would produce an increased relative radiodensity, whereas BFR mainly affecting the cortex would decrease the relative radiodensity. In addition, many different types of BFR may be prevalent in the same bone flap.

\section{Radiological Considerations}

Because the radiological method used for RBV evaluation requires no subjective decisions, the extent of BFR is objectively described by the RBV results within the limits of measurement accuracy. The major weakness concerning the volumetric method used here is the partial volume effect in some areas of the cranium. This effect could be reduced by using only $\mathrm{CT}$ scans with a very thin slice thickness, preferably less than $1.0 \mathrm{~mm}$.

In a recent study by Srivastava et al. assessing the effect of slice thickness on CT volumetry accuracy, an error of $2 \%-3 \%$ was reported with $10-\mathrm{mm}$-thick CT images when measuring a volume of $100 \mathrm{~cm} .{ }^{3,40}$ However, the measurements presented here were performed with a vertical space 

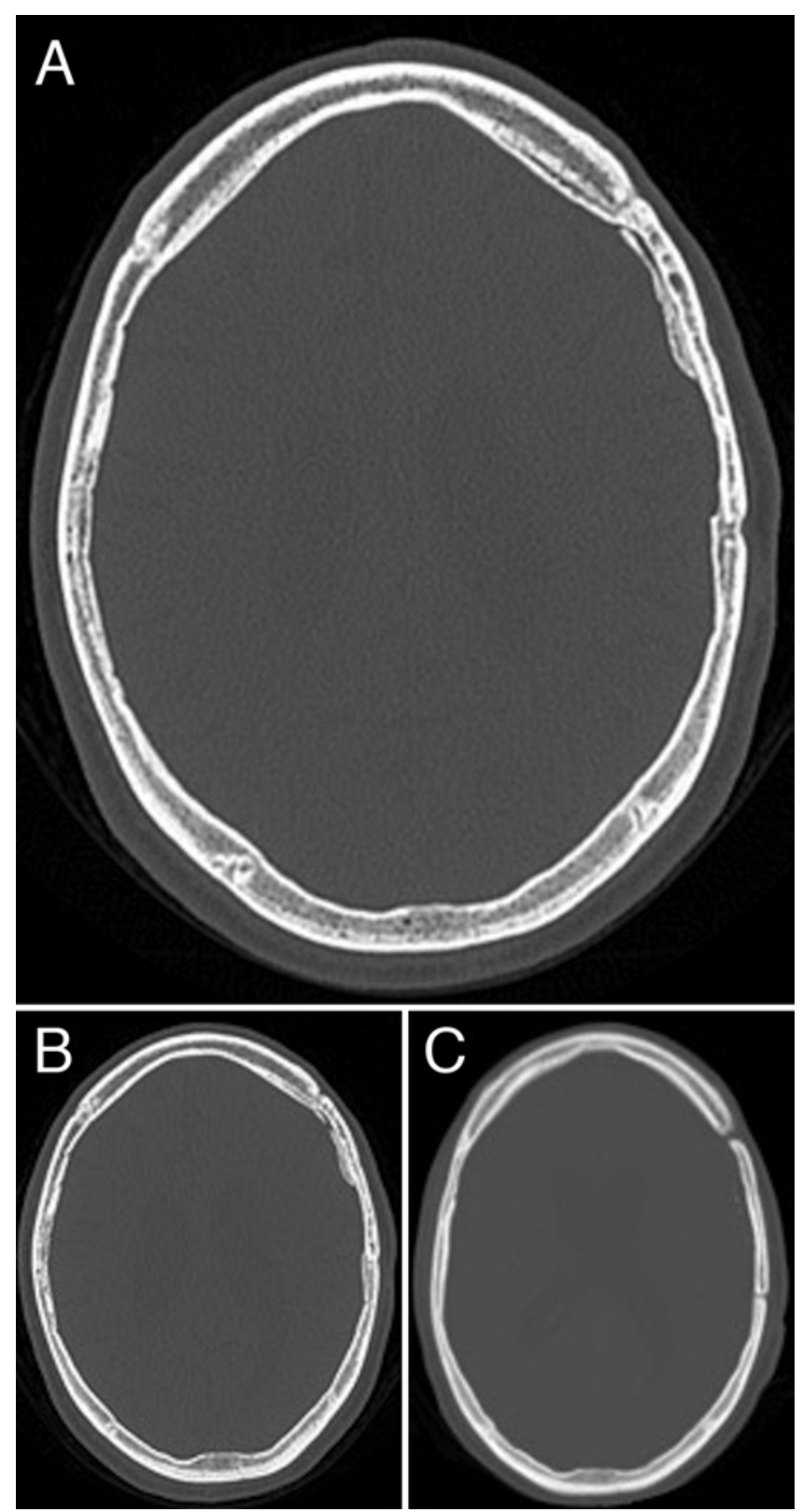

FIG. 5. Axial views of the bone flap in the 28 -year-old male patient with the longest follow-up time (132.1 months) and also the highest RBV (103.8\%). The intact bone flap CT scan (C) shows that the flap has not yet reintegrated into the cranium. In the follow-up views (A and B), however, the gaps between the bone flap and the cranium have closed, and even some osteogenesis is seen on the inner surface of the bone flap. Despite the excellent clinical outcome, the radiologist noted progressive focal resorption behind a fixation plate in the anterosuperior part of the bone flap.

(as distinguished from slice thickness) of $10 \mathrm{~mm}$ between the CT slices used for volumetry, which may decrease the partial volume effect inherent in CT volumetry. In addition, any potential over- or underestimation artifact was further minimized using our method by recording relative changes in bone flap volume and radiodensity instead of absolute values.

Intraobserver variability was reduced in the study by avoiding lengthy measurement sessions. However, because no interobserver variability was involved, we were unable to test for this variable, as would be necessary to assess the generalizability of the method. Concerning various skeletal sites, however, interobserver variability in CT volumetry has been described to be nonsignificant. ${ }^{8}$

\section{Strengths and Weaknesses}

The strength of this work lies in the fact that we were able to identify all the patients with autologous cranioplasty, and that only 4 patients were lost during follow-up. If radiological data were missing, we were able to query the secondary centers in our district for further information to ensure that the maximum number of patients could be included. In the end, only $8.9 \%$ of the original autologous cranioplasty patients were missed. Thus, the conclusions drawn in this paper are accurately based on the whole cohort. In addition, the follow-up period was relatively long.

The absolute cutoff age for performing emergency decompressive craniectomy in our institution is 65 years, so the cohort did not include any patients over that age. Similarly, the conclusions could not be extended to children under 15 years of age either. It is also inherently possible that a set of patients who have suffered from an illness critical enough to warrant decompressive surgery may well include cases in which the clinical signs of BFR have been underestimated due to impaired cognition and communication or low motivation for additional surgery. Also, all the weaknesses associated with the retrospective study design apply in the present case, as the clinical variables were gathered retrospectively.

\section{Conclusions}

BFR turned out to be a very common phenomenon, as $90 \%$ of our patients had at least some degree of resorption. Decreases in RBV were especially prominent in patients younger than 30 years of age. The progression of resorption was shown in the follow-up to be nonlinear, however. Our findings suggest that routine follow-up CT scans of all patients to monitor BFR are not necessary. Instead, clinical follow-up with mechanical stability assessment is advised. Minor resorption is most likely a normal physiological phenomenon during the bone revitalization process.

\section{References}

1. Azoulay D, Castaing D, Smail A, Adam R, Cailliez V, Laurent A, et al: Resection of nonresectable liver metastases from colorectal cancer after percutaneous portal vein embolization. Ann Surg 231:480-486, 2000

2. Balakrishnan C, Vashi C, Jackson O, Hess J: Post-traumatic osteomyelitis of the clavicle: a case report and review of literature. Can J Plast Surg 16:89-91, 2008

3. Bhaskar IP, Yusheng L, Zheng M, Lee GYF: Autogenous skull flaps stored frozen for more than 6 months: do they remain viable? J Clin Neurosci 18:1690-1693, 2011

4. Bhaskar IP, Zaw NN, Zheng M, Lee GY: Bone flap storage following craniectomy: a survey of practices in major Australian neurosurgical centres. ANZ J Surg 81:137-141, 2011

5. Birnbaum BA, Hindman N, Lee J, Babb JS: Multi-detector row $\mathrm{CT}$ attenuation measurements: assessment of intra- and interscanner variability with an anthropomorphic body CT phantom. Radiology 242:109-119, 2007 
6. Bowers CA, Riva-Cambrin J, Hertzler DA II, Walker ML: Risk factors and rates of bone flap resorption in pediatric patients after decompressive craniectomy for traumatic brain injury. J Neurosurg Pediatr 11:526-532, 2013

7. Breiman RS, Beck JW, Korobkin M, Glenny R, Akwari OE, Heaston DK, et al: Volume determinations using computed tomography. AJR Am J Roentgenol 138:329-333, 1982

8. Brindle JM, Trindade AA, Pichardo JC, Myers SL, Shah AP, Bolch WE: CT volumetry of the skeletal tissues. Med Phys 33:3796-3803, 2006

9. Brommeland T, Rydning PN, Pripp AH, Helseth E: Cranioplasty complications and risk factors associated with bone flap resorption. Scand J Trauma Resusc Emerg Med 23:75, 2015

10. Di Stefano C, Rinaldesi ML, Quinquinio C, Ridolfi C, Vallasciani M, Sturiale C, et al: Neuropsychological changes and cranioplasty: A group analysis. Brain Inj 30:164-171, 2016

11. Dünisch P, Walter J, Sakr Y, Kalff R, Waschke A, Ewald C: Risk factors of aseptic bone resorption: a study after autologous bone flap reinsertion due to decompressive craniotomy. J Neurosurg 118:1141-1147, 2013

12. Fung C, Murek M, Z'Graggen WJ, Krähenbühl AK, Gautschi OP, Schucht $P$, et al: Decompressive hemicraniectomy in patients with supratentorial intracerebral hemorrhage. Stroke 43:3207-3211, 2012

13. Geraghty EM, Boone JM, McGahan JP, Jain K: Normal organ volume assessment from abdominal CT. Abdom Imaging 29:482-490, 2004

14. Goldstein JA, Paliga JT, Bartlett SP: Cranioplasty: indications and advances. Curr Opin Otolaryngol Head Neck Surg 21:400-409, 2013

15. Goodrich JT, Sandler AL, Tepper O: A review of reconstructive materials for use in craniofacial surgery bone fixation materials, bone substitutes, and distractors. Childs Nerv Syst 28:1577-1588, 2012

16. Grant GA, Jolley M, Ellenbogen RG, Roberts TS, Gruss JR, Loeser JD: Failure of autologous bone-assisted cranioplasty following decompressive craniectomy in children and adolescents. J Neurosurg 100 (2 Suppl Pediatrics):163-168, 2004

17. Halani SH, Chu JK, Malcolm JG, Rindler RS, Allen JW, Grossberg JA, Pradilla G, Ahmad FU: Effects of cranioplasty on cerebral blood flow following decompressive craniectomy: a systematic review of the literature. Neurosurgery 81:204216,2017

18. Heymsfield SB, Fulenwider T, Nordlinger B, Barlow R, Sones P, Kutner M: Accurate measurement of liver, kidney, and spleen volume and mass by computerized axial tomography. Ann Intern Med 90:185-187, 1979

19. Honeybul S, Morrison DA, Ho KM, Lind CRP, Geelhoed E: A randomized controlled trial comparing autologous cranioplasty with custom-made titanium cranioplasty. J Neurosurg 126:81-90, 2017

20. Hutchinson PJ, Kolias AG, Timofeev IS, Corteen EA, Czosnyka M, Timothy J, et al: Trial of decompressive craniectomy for traumatic intracranial hypertension. N Engl J Med 375:1119-1130, 2016

21. Klinger DR, Madden C, Beshay J, White J, Gambrell K, Rickert K: Autologous and acrylic cranioplasty: a review of 10 years and 258 cases. World Neurosurg 82:e525-e530, 2014

22. Lam S, Kuether J, Fong A, Reid R: Cranioplasty for largesized calvarial defects in the pediatric population: a review. Craniomaxillofac Trauma Reconstr 8:159-170, 2015

23. Lemke AJ, Brinkmann MJ, Schott T, Niehues SM, Settmacher U, Neuhaus P, et al: Living donor right liver lobes: preoperative CT volumetric measurement for calculation of intraoperative weight and volume. Radiology 240:736-742, 2006
24. Levi C, Gray JE, McCullough EC, Hattery RR: The unreliability of CT numbers as absolute values. AJR Am J Roentgenol 139:443-447, 1982

25. Lin AY, Kinsella CR Jr, Rottgers SA, Smith DM, Grunwaldt LJ, Cooper GM, et al: Custom porous polyethylene implants for large-scale pediatric skull reconstruction: early outcomes. J Craniofac Surg 23:67-70, 2012

26. Martin KD, Franz B, Kirsch M, Polanski W, von der Hagen M, Schackert G, et al: Autologous bone flap cranioplasty following decompressive craniectomy is combined with a high complication rate in pediatric traumatic brain injury patients. Acta Neurochir (Wien) 156:813-824, 2014

27. Mikami T, Miyata K, Komatsu K, Yamashita K, Wanibuchi M, Mikuni N: Exposure of titanium implants after cranioplasty: A matter of long-term consequences. Interdiscip Neurosurg 8:64-67, 2017

28. Moreira-Gonzalez A, Jackson IT, Miyawaki T, Barakat K, DiNick V: Clinical outcome in cranioplasty: critical review in long-term follow-up. J Craniofac Surg 14:144-153, 2003

29. Moser T, Ehlinger M, Chelli Bouaziz M, Fethi Ladeb M, Durckel J, Dosch JC: Pitfalls in osteoarticular imaging: how to distinguish bone infection from tumour? Diagn Interv Imaging 93:351-359, 2012

30. Mustroph CM, Malcolm JG, Rindler RS, Chu JK, Grossberg JA, Pradilla G, et al: Cranioplasty infection and resorption are associated with presence of a ventriculoperitoneal shunt: a systematic review and meta-analysis. World Neurosurg 103:686-693, 2017

31. Oh JH, Zöller JE, Kübler A: A new bone banking technique to maintain osteoblast viability in frozen human iliac cancellous bone. Cryobiology 44:279-287, 2002

32. Piedra MP, Nemecek AN, Ragel BT: Timing of cranioplasty after decompressive craniectomy for trauma. Surg Neurol Int 5:25, 2014

33. Piedra MP, Thompson EM, Selden NR, Ragel BT, Guillaume DJ: Optimal timing of autologous cranioplasty after decompressive craniectomy in children. J Neurosurg Pediatr 10:268-272, 2012

34. Sarov M, Guichard JP, Chibarro S, Guettard E, Godin O, Yelnik A, et al: Sinking skin flap syndrome and paradoxical herniation after hemicraniectomy for malignant hemispheric infarction. Stroke 41:560-562, 2010

35. Schuss P, Vatter H, Oszvald A, Marquardt G, Imöhl L, Seifert $\mathrm{V}$, et al: Bone flap resorption: risk factors for the development of a long-term complication following cranioplasty after decompressive craniectomy. J Neurotrauma 30:91-95, 2013

36. Schwarz F, Dünisch P, Walter J, Sakr Y, Kalff R, Ewald C: Cranioplasty after decompressive craniectomy: is there a rationale for an initial artificial bone-substitute implant? A single-center experience after 631 procedures. J Neurosurg 124:710-715, 2016

37. Shah AM, Jung H, Skirboll S: Materials used in cranioplasty: a history and analysis. Neurosurg Focus 36(4):E19, 2014

38. Shahid AH, Mohanty M, Singla N, Mittal BR, Gupta SK: The effect of cranioplasty following decompressive craniectomy on cerebral blood perfusion, neurological, and cognitive outcome. J Neurosurg 128:229-235, 2018

39. Sohaib SA, Turner B, Hanson JA, Farquharson M, Oliver RT, Reznek RH: CT assessment of tumour response to treatment: comparison of linear, cross-sectional and volumetric measures of tumour size. Br J Radiol 73:1178-1184, 2000

40. Srivastava SP, Cheng CW, Das IJ: The effect of slice thickness on target and organs at risk volumes, dosimetric coverage and radiobiological impact in IMRT planning. Clin Transl Oncol 18:469-479, 2016

41. Stieglitz LH, Fung C, Murek M, Fichtner J, Raabe A, Beck $\mathrm{J}$ : What happens to the bone flap? Long-term outcome after reimplantation of cryoconserved bone flaps in a consecutive 
series of 92 patients. Acta Neurochir (Wien) 157:275-280, 2015

42. Vahedi K, Hofmeijer J, Juettler E, Vicaut E, George B, Algra A, et al: Early decompressive surgery in malignant infarction of the middle cerebral artery: a pooled analysis of three randomised controlled trials. Lancet Neurol 6:215-222, 2007

43. Zanaty M, Chalouhi N, Starke RM, Clark SW, Bovenzi CD, Saigh M, et al: Complications following cranioplasty: incidence and predictors in 348 cases. J Neurosurg 123:182188,2015

44. Zanotti B, Zingaretti N, Verlicchi A, Robiony M, Alfieri A, Parodi PC: Cranioplasty: review of materials. J Craniofac Surg 27:2061-2072, 2016

45. Zhang J, Peng F, Liu Z, Luan J, Liu X, Fei C, et al: Cranioplasty with autogenous bone flaps cryopreserved in povidone iodine: a long-term follow-up study. J Neurosurg 127:14491456, 2017

\section{Disclosures}

The authors report no conflict of interest concerning the materials or methods used in this study or the findings specified in this paper.

\section{Author Contributions}

Conception and design: all authors. Acquisition of data: Korhonen, Salokorpi, Niinimäki, Tetri. Analysis and interpretation of data: all authors. Drafting the article: all authors. Critically revising the article: all authors. Reviewed submitted version of manuscript: all authors. Approved the final version of the manuscript on behalf of all authors: Korhonen. Statistical analysis: Korhonen, Salokorpi, Tetri. Study supervision: Serlo, Lehenkari, Tetri.

\section{Correspondence}

Tommi Korhonen: Oulu University Hospital, Oulu, Finland. tommi.korhonen@student.oulu.fi. 